\title{
Buffered local anaesthetic and injection pain and onset time
}

\section{Abstracted from}

Aulestia-Viera PV, Braga MM, Borsatti MA.

The effect of adjusting the $\mathrm{pH}$ of local anaesthetics in dentistry: a systematic review and meta-analysis. Int Endod / 2018; 51: 862-876. doi: 10.1111/iej.12899. [Epub ahead of print] Review. PubMed PMID: 29377171.

Address for correspondence: Department of Stomatology, School of Dentistry, University of São Paulo, São Paulo, Brazil.

\section{Question: Does buffered local anaesthetic reduce infiltration pain and onset time during dental procedures?}

Data sources Medline, Embase, Scopus and Scielo were searched until April 2017 supplemented by manual searching.

Study selection Clinical trials with parallel-groups, crossover and split mouth in patients older than 18 years were included with no language restrictions. The primary outcome considered was changes in pain intensity, while the secondary outcome was changes on onset time. Data extraction and synthesis Two authors extracted data using a standard data form. Inter-rater reliability was calculated as high. For the primary outcome all scales were converted to a 0-100 scale. For the secondary outcome all measures were normalised in minutes. Risk of bias was assessed using the Cochrane Collaboration tool. The treatment effect was calculated using mean differences and $95 \%$ confidence interval. Heterogeneity and sensitivity analysis were performed. Data were combined using a random effect model. Results Fourteen studies were included in the review. Of those nine were included in a meta-analysis for the primary outcome pain showing no significant reduction: -7.38 (-15.67-0.91) for normal tissue (five studies) and -4.41 (-15.25-6.43) for inflamed tissue on four studies. No statistically significant results were found in the block (three studies) or the infiltration groups (seven studies). Four studies were included in a meta-analysis for onset time. Significant reduction was achieved for inferior alveolar nerve blocks: $-1.26(-1.41$ to -1.10$)$ on three studies and when used on inflamed tissue: $-1.37(-2.03$ to -0.70 ) from one study. High degree of heterogeneity was observed in all groups.

Conclusions The authors concluded that buffered lidocaine does not reduce pain from injection and the onset time reduction in inferior alveolar nerve blocks and in inflamed tissues while the magnitude of the reduction is not clinically relevant.

\section{Commentary}

Pain during injection of a local anaesthetic may profoundly affect a patient's experience. Current local anaesthetic agents used for injection are weak bases packed in an acidic formulation to increase stability and shelf life. The low $\mathrm{pH}$, usually below five, is in part responsible for the pain during injection and slow onset.

This review summarises the evidence on the effect on the use of buffered anaesthetic agent, compared to unbuffered, on pain and onset. Risk of bias was assessed as low in four studies and high in three, which were later excluded from the metanalysis in addition to the two non-lidocaine studies.

From the included studies 12 used lidocaine, one bupivacaine and one articaine. From the lidocaine studies: 2\% w/epinephrine was used in 11 studies (w/epi 1:100.00 and two w/epi 1:80.000) while $4 \%$ w/epinephrine 1:100.000 was used in two studies. Eight studies were cross over or split mouth (ranging between 80 to 18 participants in each) and the rest were parallel design (ranging between 100 to 40 participants in each). It is unclear if power calculation was performed in any of the included studies or the magnitude of the difference that was considered significant since previous studies established the minimum perceptible difference in pain to be 15 on a $0-100$ scale.

Because of the use of different pain scales the authors converted all into a 0-100 scale, a practice based on a previous systematic review on the effect of warming the anaesthetic agent on pain, although that review did not include studies assessing pain on a four point scale such as this one. Other variabilities in the included studies relate to the use of topical anaesthetics (five studies), differences on the ways of assessing onset (soft tissue, EPT), type of injection, tissue condition, temperature of the anaesthetic, needle size and speed of injection. Subgroup analysis was performed for normal versus inflamed tissue, and block versus infiltration. The reported statistically significant results were based on single studies.

The authors concluded that the high degree of heterogeneity observed was based on the use of different pain scales and as such should be taken into consideration for future trials. Since buffering takes place immediately before injection, changes on the time of onset may not be relevant to the practitioner and that is not even considering the extra cost.

Silvia Spivakovsky New York University, College of Dentistry, New York, USA 